\title{
Evaluation of a bundled care approach to preventing surgical site infections post caesarean section in an Australian Rural Hospital
}

\section{Michelle Bolte}

Tamworth Rural Referral Hospital

\section{Blake Knapman}

Wollongong Hospital

Lilach Leibenson

Tamworth Rural Referral Hospital

Jean Ball

Hunter New England Local Health District https://orcid.org/0000-0001-5402-6415

Michelle Giles ( $\square$ Michelle.Giles@health.nsw.gov.au )

https://orcid.org/0000-0002-8611-7941

Research article

Keywords: Caesarean, surgical site infection, infection prevention bundle, bundled approach

Posted Date: July 30th, 2019

DOI: https://doi.org/10.21203/rs.2.12111/v1

License: (9) This work is licensed under a Creative Commons Attribution 4.0 International License.

Read Full License 


\section{Abstract}

Background Elective and non-elective caesarean section (CS) rates have been increasing in Australia over the past 20 years. Increasing antenatal morbidity, has meant that surgical site infection (SSI) post CS is an important issue effecting Australian women. Populations most impacted include low socioeconomic and regional communities where high rates of antenatal comorbidity increase the incidence of SSI.

Despite a recent trend towards supporting the development of evidence based bundled approaches to SSI reduction, there remains a paucity of data proving efficacy and supporting bundle implementation. Aims This study aimed to develop, implement and assess an evidence based caesarean infection prevention "CIP" bundled intervention to reduce SSI rates following CS in a high risk rural population. Methods The study was a pre-post-intervention study with 3 phases and included all women undergoing CS at a regional referral hospital between December 1st 2016 and December 31st 2018. A 12 month retrospective pre-intervention review identified all women who developed a post CS SSI. A comprehensive literature review informed the development of the intervention, which was implemented in December 2017. Prospective data was collected for a subsequent 12 month period on all women who underwent CS with pre and post comparative data analysis. Results A total of 710 procedures were monitored as part of the study with 346 and 364 women in the pre and post-intervention groups respectively. Demographic and comorbidity variables remained consistent between the two time periods. Rates of CS associated SSI reduced significantly post-intervention ( $5.5 \%$ vs. $1.6 \%, p=0.007)$, the greatest benefit seen in class II and III obese patients $(12.2 \%$ vs. $2.5 \%, p=0.019)$. Higher rates of hypertension ( $24 \%$ vs. $9 \%, p=0.01)$ and lower maternal age (mean age 27 vs. 30, p=0.01) were seen in patients with SSI. Rates of smoking were higher in women effected by SSI (24\% vs $9 \%$ ) but did not reach statistical significance. Conclusion The "CIP" bundle is an effective method of reducing caesarean associated surgical site infections in a high risk population. Our findings highlight the necessity for the development and evaluation of multifaceted, evidenced based interventions to reduce SSI in women requiring caesarean delivery.

\section{Background}

Caesarean section (CS) is a commonly performed obstetric procedure [1]. In 2017, 33.8\% of women in NSW required operative delivery, representing an increase of 2.3\% since 2013 [2-6]. Increasing rates of intervention, combined with increasing obstetric patient comorbidity, are increasing rates of CS associated surgical site infections (SSI). In Australia, SSIs are the third most common nosocomial infection [7]. Voluntary data from the Australasian Clinical Indicator Report indicates post CS SSI rates of $0.5 \%$ across Australia and New Zealand [8]. This is in contrast to global rates which range from 3-15\% [9] and likely reflect under-reporting of post CS SSI as a consequence of earlier discharges from hospital, varied definitions, post-operative surveillance tools and community health management [1]. It is estimated that $90-95 \%$ of SSIs post CS occur post discharge from initial hospitalisation [10-12]. Avoiding SSI is an important healthcare issue as sepsis remains a leading cause of maternal death in Australia and the United Kingdom [13, 14]. 
Patients that experience SSI report significant physical, emotional, and economic cost culminating in social isolation $[9,15]$. As a consequence post CS SSIs are associated with substantial psychosocial and economic burden to mothers and the healthcare system. International econometric analysis suggests statistically significant increases in health department costs associated with prolonged hospital admission, pharmaceuticals and laboratory investigations [16].

Infection risk assessment is an important preliminary step in identifying women predisposed to SSI, and implementation of effective measures to improve wound healing and prevent infection is vital [12].

Documented risk factors including obesity, smoking, diabetes, chorioamnionitis and hypertension already inform standard risk reducing recommendations in the antenatal, intrapartum and postnatal settings [1719]. Other factors that impact on women's risk of developing SSI post CS include the length of surgery, use of prophylactic antibiotics, type of skin preparation and type of skin closure technique [20].

In the past, the literature has focused on single intervention risk reducing recommendations that aim to modify these factors and are readily accessible to clinicians $[6,21]$. Recent interest in bundled prevention strategies has stimulated the publication of systematic reviews assessing individual best practice aspects and, in doing so, developing CS SSI prevention suites [22]. Despite this, there is a paucity of evidence supporting bundle implementation. This study aims to assess the impact of an evidence based bundled infection prevention strategy, referred to as the "Caesarean Infection Prevention Bundle" ("CIP"), in reducing rates of SSI in women following CS at a rural referral hospital in New South Wales, Australia.

\section{Methods}

This was a pre-post control-intervention study with a phased approach conducted over a two year period from December 2016 through to December 2018. The study was conducted in a rural acute care hospital within a NSW Local Health District. The hospital is categorised as rural in accordance with the Rural, Remote and Metropolitan Areas Classification [23] and has approximately 1000 births annually, with $36 \%$ via CS. The population is an unselected cohort including all women undergoing CS between the $1^{\text {st }}$ of December 2016 and the $31^{\text {st }}$ of December 2018. Consent was not required as all data used was routinely collected as part of inpatient care.

Phase 1 of the study involved pre-intervention data collection, a retrospective review of all patients presenting to the emergency department or requiring readmission to the study hospital or other rural hospital with a CS associated SSI between December 2016 and December 2017.

\section{SSI Surveillance}

SSIs were identified using a multifaceted process including review of medical record of all women undergoing CS, emergency department encounters and hospital readmissions for wound complications within a 30 day post-operative period. In addition, infection related events within the 30 day post- 
operative period, in an already established Health Service Infection Control database were identified. Strict adherence to Centre of Disease Control (CDC) diagnostic criteria were required for inclusion [24].

Cases meeting inclusion criteria were reviewed for demographics such as age, body mass index (BMI), and pregnancy related risk factors including smoking, hypertension, diabetes and chorioamnionitis. CS related data collected included surgical time, the indication for CS, booking category and use of prophylactic antibiotics. Analysis identified a CS associated SSI rate of 5.5\% during the 12 month pre audit period. This pre-intervention data demonstrated obesity as the greatest predictor of infection with additional risk factors including smoking and diabetes.

Phase 2 involved the development and implementation of the "CIP" bundle (Table 1). A comprehensive literature review was performed identifying and evaluating five individual infection reduction strategies. The "CIP" bundle represents a synthesis of these findings and was developed in consultation with relevant expert stakeholders.

\section{Implementation}

Implementation was achieved using evidence based strategies focusing on extensive collaboration, education [25], feedback [26] and the development of intervention resources [27]. The multidisciplinary team involved senior clinicians from obstetrics, midwifery, nursing and anaesthetics. Education was delivered [25] to all relevant stakeholders by the Infection Prevention and Control Clinical Nurse Consultant (CNC) and four nominated ward and operating suite based Clinical Nurse Educators. Data monitoring and regular interval feedback was provided focusing on bundle compliance and evolving SSI rates to encourage protocol adherence [26]. Resources were developed, including flyers, information sheets and posters [27]. These were distributed to relevant staff and displayed in clinical areas.

Women at high risk of SSI were identified during the antenatal period in accordance with a risk assessment algorithm developed, based on an extensive literature review and pre audit data findings (Figure 1). Women presenting for elective CS washed at the facility using plain soap on the morning of their procedure before changing into a hospital gown. In the instance of an emergency procedure, neutral detergent bed bath wipes were used by midwifery staff while preparing for operative management. Antibiotic prophylaxis was administered prior to skin incision in accordance with local and international recommendations $[3,6,28]$. Prophylaxis timing of 15 to- 60 minutes prior to skin incision was used to achieve optimal tissue saturation prior to skin incision $[28,29]$. Chlorhexidine $2 \%$ in isopropyl $70 \%$ alcohol was used for skin preparation in all CSs with a mandatory latency period prior to surgical draping to allow drying and prevent spontaneous combustion [6].

Figure 1: "CIP" Bundle Risk Assessment Algorithm

In cases of category $1 \mathrm{CS}$ [30], aqueous povidone-iodine was used to facilitate rapid abdominal preparation and entry. Post-operatively, negative pressure wound therapy (NPWT) dressings were used on 
all women with a $\mathrm{BMI} \geq 35$ (class II \& III obese), those with diabetes and women that smoked during pregnancy and were thus considered high risk for SSI [6,31]. The type of NPWT dressing used was determined by BMI with class II and class III obese women receiving the PICO ${ }^{\mathrm{TM}}$ [32] and Prevena [33] NPWT dressings respectively. This stratified approach was employed to enhance cost effectiveness with the more expensive Prevena system, which is larger and provides greater negative pressure $(125 \mathrm{mmHg}$ vs $80 \mathrm{mmHg}$ ), being reserved for women with larger subcuticular tissue [32, 33]. Negative pressure dressings remained in-situ for 7 days and were removed by home maternity services or community nurses depending on the woman's location. Bundle implementation occurred over a four week period in December 2017. A summary of the bundle and corresponding evidence can be found in table 1 .

Phase 3 of the study included prospective post-intervention data collection and analysis. The primary outcome was the rate of SSIs post CS, identified as per the surveillance process used in phase one. Case demographics were collected in alignment with phase 1 of the study. Secondary outcomes included bundle adherence and intervention impact stratified for patient demographics and adherence to individual infection prevention strategies within the bundle.

\section{Data analysis}

Univariate analyses were conducted to assess any differences in the populations of interest at preintervention and post-intervention. Univariate analyses were also conducted to test for differences in demographic and comorbidity variables of interest, between those with a SSI and those without. Demographic and comorbidity variables were defined as maternal age, BMI, smoking status, diabetes and hypertension.

CS and SSI rates were compared pre and post-intervention using t-tests and logistic regression models. Exact analyses were used where there were small numbers of observations.

To test for a difference in SSI rates pre and post-intervention, a multivariable model was constructed, which included the demographic and comorbidity variables of interest. The multivariable model was assessed for variables which could be removed without affecting the model and a final multivariable model was constructed.

Compliance with bundle elements was monitored for all women delivering by CS in the post-intervention period and proportions aggregated monthly.

\section{Results}

There were 1,002 births in the pre-intervention phase and 929 births in the post-intervention phase. Of these births, 346 (35\%) were by CS in the pre-intervention phase and 364 (39\%) were by CS in the postintervention phase. See Table 2. 
There were 19 SSIs in the pre-intervention phase, 11 of these were in women with a $\mathrm{BMI} \geq 35$, giving an overall infection rate of $5.5 \%$ and an infection rate of $12.2 \%$ in the $\mathrm{BMI} \geq 35$ group. Post-intervention, there were 6 SSIs, 2 of which were in the BMI $\geq 35$ group, giving infection rates of $1.6 \%$ and $2.5 \%$ respectively. See Table 2.

Univariate analysis (without adjusting for other factors) showed a significant decrease in overall SSI rates from pre-intervention to post-intervention $(\mathrm{p}=0.007)$. The pre-intervention $\mathrm{SSI}$ rate in the $\mathrm{BMI} \geq 35$ group significantly decreased post-intervention $(\mathrm{OR}[95 \% \mathrm{Cl}]=0.15[0.03,0.73] ; \mathrm{p}=0.019)$.

The demographic and comorbidity variables (maternal age, BMl, smoking status, diabetes and hypertension) each showed no significant difference pre and post-intervention, illustrating no difference in the populations at pre-intervention and post-intervention. See Tables 3 and 4 . In particular, there were 21 CS patients who were aged $<20$ years $(6 \%)$ in the pre-intervention phase, and of these, $3(14 \%$ of all SSIs) had an SSI. At post-intervention, $24(7 \%)$ of patients were $<20$ years of age with the SSI prevalence reducing to $8 \%$ (2 patients) of all SSIs (Table 4 ).

Table 5 shows the pre-intervention comparison between SSI groups for demographic and comorbidity variables for all CS.

Maternal age was significantly lower in the SSI group when compared to the no SSI group (mean age for those without SSI was 30 and for those with SSI was 27; $=0.03$ ).

BMI was significantly higher in the SSI group when compared to the no SSI group (mean BMI for those without SSI was 29 and for those with SSI was $36 ; p<0.01$ ).

Hypertension was significantly higher in the SSI group when compared to the no SSI group (prevalence for those without SSI was $9 \%$ and for those with SSI was $24 \%$; $p=0.02$ ).

Smoking was non-significantly higher in the SSI group when compared to the no SSI group (prevalence of smokers was $21 \%$ and $36 \%$ in the no SSI group and the SSI group respectively).

There were no differences in demographic and comorbidity variables between patients with and patients without an SSI for those with BMI>=35.

A logistic regression model was constructed with SSI as the outcome and included a pre-post-intervention variable, maternal age, BMI, smoking status, diabetes and hypertension. Diabetes was removed without affecting any other variables in the model. There was a significant decrease in SSI post-intervention $(\mathrm{OR}=0.31[0.12,0.80], \mathrm{p}=0.016)$. Maternal age was significantly lower in the SSI group post-intervention $(\mathrm{OR}=0.91[0.84,0.99], \mathrm{prob}=0.02)$ and $\mathrm{BMI}$ was significantly higher in the SSI group post-intervention $(\mathrm{OR}=1.11[1.05,1.16]$, prob<0.0001). See Table 6 , which shows results for the univariate (unadjusted) model and the multivariable (adjusted) model. 
For the high risk group (BMl>=35), a similar significant decrease was evident in the post-intervention group $(\mathrm{OR}=0.15[0.03,0.73], \mathrm{p}=0.019)$, as shown in Table 7.

\section{Practice Adherence}

Bundle practice adherence was assessed on all CS post-intervention for 12 months. Compliance with preoperative site skin preparation hygiene prior to surgery increased over the 12 month post-implementation period (Figure 2). Although documentation was poor initially, when staff were asked about the practice they confirmed that it was being done. The introduction of a sticker stating bed bath wipe/shower to the pre-operative checklist saw an improvement in documentation (See Figure 2).

Figure 2: Percent of patients who received pre-operative site skin preparation

Pre-operative skin preparation with chlorhexidine and alcohol improved over the 12 month postimplementation period and as surgeons became more comfortable with its use, adherence increased (Figure 3). Women who underwent an emergency CS were excluded from the adherence auditing.

Figure 3: Percent of patients who received operative site preparation with Chlorhexidine and alcohol Figure 4 demonstrates prophylaxis antibiotic administration adherence 15 to 30 minutes before incision time over the 12 month post-implementation period. As adherence increased over time, infection rates decreased. Feedback on practice adherence and educational support was given regularly to the obstetric teams during the data collection process.

Negative pressure dressings for post-operative management were used on all high risk patients identified in the bundle. However, the type of dressing (PICO for BMI 35 to 40 and Prevena for BMI $>40$ ) was not consistently adhered to during the early months post implementation. Compliance with protocol improved over time. By 8 months post-intervention, 100\% adherence to protocol was achieved, through ongoing feedback and educational support.

Figure 4: Percent of patients who received antibiotics prophylaxis 15-60 minutes pre-incision

\section{Discussion}

This project identified a statistically significant reduction in SSI rates with the introduction of the "CIP" bundle (5.5\% vs. $1.6 \%, \mathrm{p}=0.007)$. The impact of the bundle was most marked in those categorized as high risk in the antenatal period. When stratified for Class II and III obesity, the data demonstrates a reduction from $12.2 \%$ to $2.5 \%$ in women with a $B M I \geq 35$ ( $p=0.019)$. This group represents $22 \%$ of included cases and thus illustrates a significant reduction in post-operative SSI within this high risk demographic. Further subset analysis demonstrated rates of infection among class III obese patients, who comprise $9 \%$ of the 
studied population, fell significantly from $15.4 \%$ to $5.9 \%$ post-intervention. Comparison to existing data illustrates bundle efficacy with instances of SSI falling below previously documented rates in obese patients [44]. Despite this, obesity remains a significant risk factor for SSI in the post-intervention group with $33 \%$ of infections occurring in obese patients.

Comparison of instances of SSI within the study demonstrates common complicit factors. Those representing with infection post-intervention were more likely to be emergency procedures with only two of the six identified SSIs found in cases of elective CS. This is congruent with existing data that consistently shows increased infection rates in emergency procedures $[38,45]$. Despite each patient having recognised risk factors, bundle compliance was reduced in both encounters. Of note, the selected negative pressure dressings were removed inappropriately early, potentially reducing the efficacy of the intervention. In addition, documentation regarding reasons for removal is poor. This highlights the need for continued staff education in dressing application and post-operative management. Review of data collected on negative pressure dressing removal shows that early removal occurred in $8 \%$ of cases, the majority of these occurring early in the intervention phase of the study, with adherence improving over time.

Antibiotic administration targets were not met in all cases of post-intervention SSI. Best practice dictates antibiotic administration occurs 15-60 minutes prior to skin incision to allow tissue saturation at the time of operative management $[28,29]$. Of those encounters complicated by SSI, $50 \%$ had pre-incision administration, however none occurred within the ideal operative window. Although antibiotic compliance improved over time, achieving uniformity in practice has been a significant challenge. This is compounded by suboptimal documentation of administrative times with $50 \%$ of those treated for SSI having no recorded time of antibiotic administration.

As anticipated, bundle compliance improved significantly over the 12 month study period. In the first six months, ideal antibiotic administration occurred in $18 \%$ of encounters. This increased to $78 \%$ in the final two months of the intervention period. Documentation of antibiotic administration times also significantly improved, trending to $100 \%$ accuracy over the same period. This marked increase in compliance is attributed to ongoing multidisciplinary education sessions where key performance indicators were presented and discussed with relevant stakeholders, in particular anaesthetists. Compliance barriers may have been overcome earlier through direct involvement of an anaesthetist in the study group in the planning phase. Significant increases in rates of pre-operative washing, the use of chlorhexidine-alcohol skin preparation and appropriate dressing selection were also observed over the 12 month period. These increases coincide with falling rates of SSI with no infections recorded following the $8^{\text {th }}$ month of investigation.

Although the data demonstrates a significant reduction in SSI and thus supports bundle efficacy, it is not without limitation. Issues with practice adherence, particularly regarding the timing of antibiotic prophylaxis, contaminate the data and potentially underestimate the impact of the bundle. Paucity of documentation is another issue faced by investigators. This is highlighted when examining the cases of 
SSI with up to $50 \%$ of reportable data missing in each assessable domain. These rates are inconsistent with the remainder of the surgical encounters and may reflect case specific pressures not measured in the study. Although pre and post-intervention groups remain comparable, it is important to note the study's inability to capture SSIs diagnosed and managed in the community setting. Literature examining outpatient infection rates estimates that up to $90-95 \%$ of SSIs are diagnosed following hospital discharge [10-12] resulting in underestimation of true infection rates. As length of stay following CS continues to fall, multi-method post-discharge surveillance approaches will become increasingly important to monitor facility performance and promote early infection detection [46]. While the reduction in rates of SSI in this high risk population are statistically significant, rollout of the bundle across multiple sites will facilitate large volume data collection, thus achieving power, and simultaneously assess whether the intervention is transferable to a broader range of patient demographics.

\section{Conclusion}

In conclusion, this study demonstrates the efficacy of the "CIP" bundle in reducing rates of post CS SSI in a high risk population. The success of this bundled intervention supports further rigorous evaluation of multifaceted, evidenced based interventions to reduce CS associated SSI. Large scale implementation of bundled approaches may facilitate standardisation of care, improve guideline compliance, and enhance surveillance mechanisms used to monitor wound complications. As seen in previous econometric analysis, a reduction in SSI results in decreased financial burden for both healthcare systems and consumers. The combination of reduced patient morbidity, cost and psychosocial consequences mandate ongoing evaluation of a bundled approach in the reduction in CS associated SSI.

\section{Abbreviations}

BMI - Body Mass Index

CDC - Centre for Disease Control

CIP - Caesarean Infection Prevention

CNC - Clinical Nurse Consultant

CS - Caesarean Section

NPWT - Negative Pressure Wound Therapy

SSI - Surgical Site Infection

\section{Declarations}

\section{Ethics approval and consent to participate}


Ethical approval was obtained from the Hunter New England Human Research Ethics Committee $(18 / 03 / 21 / 5.11)$. Consent to participate was not applicable.

\section{Consent for publication}

Not applicable

\section{Availability of data and materials}

The datasets used and analysed during the current study are available from the corresponding author on reasonable request.

\section{Competing interests}

The authors declare that they have no competing interests.

\section{Funding}

The study was not funded and was incorporated within the daily practice of delivered care.

\section{Authors' contributions}

$M B, B K, L L$ and $M G$ contributed to the development of research conception, design and methods and have significantly critically revised and contributed to the content of this manuscript. MB and BK contributed to the initial draft of this manuscript. MB and MG contributed to data management. JB contributed to data management and analysis and contributed to the content in this manuscript. All authors have read and approved the final manuscript.

\section{Acknowledgements}

The authors would like to acknowledge Tracey Walker (Theatre Educator), Rebecca Sharpe (Midwife Educator), Lurena Smith (Midwife Educator), Dr Melissa Price-Purnell, Dr Stephanie Gorham and Robin Skillman (CNC Wound/Stoma Therapy).

The authors would also like to acknowledge the midwives, theatre nurses, resident medical officers, and other key stake holders who assisted with education, data collection and participated in implementation and sustaining the bundle practice.

\section{References}

1. Saeed K, Green R, Corcoran P, O'Neill S. Incidence of surgical site infection following caesarean section: a systematic review and meta-analysis protocol. BMJ Open. 2016;7:e013037.

2. Centre for Epidemiology and Evidence. New South Wales Mothers and Babies 2017. Sydney; 2018. 
3. Berríos-Torres S, Umscheid C, Bratzler D, Leas B, Stone E, Kelz R, et al. Centers for Disease Control and Prevention Guideline for the Prevention of Surgical Site Infection,2017. JAMA Surgery. 2017;152(8):784-91.

4. Holland C, Foster P, Ulrich D, Adkins K. A practice improvement project to reduce cesarean surgical site infection rates. Association of Women's Health, Obstetric and Neonatal Nurses. 2016;20(6):54451.

5. Webster J, Osborne S. Preoperative bathing or showering with skin antiseptics to prevent surgical site infection. Cochrane Database of Systematic Reviews. 2015(Issue 2 . Art. No.: CD004985.).

6. World Health organisation (WHO). Global guideline for the Prevention of Surgical site infections 2016 [Available from: http://www.who.int/gpsc/ssi-prevention-guidelines/en/.

7. Worth L, Bull A, Spelman T, Brett J, Richards M. Diminishing Surgical Site Infections in Australia: Time Trends in Infection Rates, Pathogens and Antimicrobial Resistance Using a Comprehensive Victorian Surveillance Program, 2002-2013. Infection Control and Hospital Epidemiology. 2015;36(4):409-16

8. Australian Council on Healthcare Standards (ACHS). Australasian Clinical Indicator Report: 19th Edition 2010-2017 An extensive review of clinical performance. Sydney; 2018.

9. Zuarez-Easton S, Zafran N, Garmi G, Salim R. Postcesarean wound infection: prevalence, impact, prevention, and management challenges. International Journal of Women's Health 2017;9 81-8.

10. Cardoso Del Monte M, Pinto Neto A. Postdischarge surveillance following cesarean section: The incidence of surgical site infection and associated factors. American Journal of Infection Control. 2010;38(6):467-72.

11. Corcoran S, Jackson V, Coulter-Smith S, Loughrey J, McKenna P, Cafferkey M. Surgical site infection after cesarean section: implementing 3 changes to improve the quality of patient care. American Journal of Infection Control 2013;41:1258-63.

12. Wloch C, Wilson J, Lamagni T, Harrington P, Charlett A, Sheridana E. Risk factors for surgical site infection following caesarean section in England: results from a multicentre cohort study. British Journal of Obstetrics and Gynaecology. 2012;119:1324-33.

13. Australian Institute of Health and Welfare (AIHW). Maternal Deaths in Australia 2016. Canberra; 2018.

14. Centre for Maternal and Child Enquiries (CMACE). Saving mothers' lives. Reviewing maternal deaths to make motherhood save: 2006-2008. BJOG: An International Journal of Obstetrics \& Gynaecology. 2011;118(Supplement 1):1402-4.

15. Andersson A, Bergh I, Karlsson J, Nilsson K. Patients' experiences of acquiring a deep surgical site infection: an interview study. American Journal of Infection Control. 2010;38(9):711-7.

16. Olsen M, Butler A, Willers D, Gross G, Hamilton B, Fraser V. Attributable costs of surgical site infection and endometritis after low transverse cesarean delivery. Infection control and hospital epidemiology. 2010;31(3):276-82. 
17. Chaim W, Bashiri A, Bar-David J, Shoham-Vardi I, Mazor M. Prevalence and Clnical Significance of Postpartum Endometritis and Wound Infection. Infections Diseases in Obstetrics and Gynecology. 2000;8:77-82.

18. Ketcheson F, Woolcott C, Allen V, Langley J. Risk factors for surgical site infection following cesarean delivery: a retrospective cohort study. CMAJ Open. 2017;5(3):E546-E56.

19. Olsen M, Butler A, Willers D, Devkota P, Gross G, Fraser V. Risk factors for surgical site infection after low transverse cesarean section. Infection Control \& Hospital Epidemiology. 2008;29(6):477-84.

20. Anderson V CW, Gillespie B. The relationship between obesity and surgical site infection in women undergoing caesarean sections:An integrative review. Midwifery. 2013:1331-8.

21. Australian Commission on Safety and Quality in Health Care (ACSQHC). Hospital -Acquired Complications Information Kit. Fact sheets to support safety and quality in Australian health services. 2018 [Available from: https://www.safetyandquality.gov.au/wpcontent/uploads/2018/03/HACs-information-kit-Introduction.pdf.

22. Martin E, Beckman M, Bransbee L, Halton K, Merollini K, Graves N. Best practice perioperative strategies and surgical techniques for preventing caesarean section surgical site infections: a systematic review if reviews and meta-analyses. Royal College of Obstetricians and Gynaecologists. 2018 125(8):956-64.

23. Australian Institute of Health and Welfare (AlHW). Rural, regional and remote health: A guide to remoteness classifications. Canberra; 2004.

24. Centre of Disease Control (CDC) Procedure-associated Module SSI 2019 [Available from: https://www.cdc.gov/nhsn/pdfs/pscmanual/9pscssicurrent.pdf.

25. Flodgren G, Conterno L, Mayhew A, Omar O, Pereira C, Shepperd S. Interventions to improve professional adherence to guidelines for prevention of device-related infections. Cochrane Database of Systematic Reviews. 2013.

26. Ivers N, Jamtvedt G, Flottorp S, Young J, Odgaard-Jensen J, French S, et al. Audit and feedback: effects on professional practice and healthcare outcomes (Review). Cochrane Database of Systematic Reviews. 2012;Issue 6. Art. No.: CD000259.

27. Giguère A, Légaré F, Grimshaw J, Turcotte S, Fiander M, Grudniewicz A, et al. Printed Educational materials: effects on profesinal practice and healthcare outcomes. Cochrane Database of Systematic Reviews. 2012(10).

28. Mackeen A, Packard R, Ota E, Berghella V, Baxter J. Timing of intravenous prophylactic antibiotics for preventing postpartum infectious morbidity in women undergoing cesarean delivery (Review). Cochrane Database of Systematic Reviews. 2014(ssue 12. Art. No.: CD009516.).

29. Royal Australian and New Zealand College of Obstetricians and Gynaecologists (RANZCOG). Prophylactic antibiotics in obstetrics and gynaecology 2016 [Available from: https://www.ranzcog.edu.au/RANZCOG_SITE/media/RANZCOGMEDIA/Women\%27s\%20Health/Statement\%20and\%20guidelines/Clinical\%20- 
\%20General/Prophylactic-antibiotics-in-obstetrics-and-gynaecology-(C-Gen-17)-Review-July2016.pdf?ext=.pdf.

30. Royal Australian and New Zealand College of Obstetricians and Gynaecologists (RANZCOG) Categorisation of urgency for caesarean section. 2015.

31. National Institute for Health and Care Excellence (NICE). PICO negative pressure wound dressings for closed surgical incisions. 2019.

32. Smith \& Nephew Global. PICO Single Use Negative Wound Therapy System 2019 [Available from: http://www.smith-nephew.com/key-products/advanced-wound-management/pico/.

33. KCl An Acelity Company. Prevena Incision Management System 2016 [Product Information Package]. Available from: https://acelity.com/healthcare-professionals/global-productcatalog/catalog/prevena-incision-management-system.

34. Ng W, Brown A, Alexander D, Ho M, Kerr B, Amato M, et al. A multifaceted prevention program to reduce infection after cesarean section: Interventions assessed using an intensive postdischarge surveillance system. American Journal of Infection Control. 2015;43:805-9.

35. Willy C, Agarwal A, Andersen C, Santis G, Gabriel A, Grauhan O, et al. Closed incision negative pressure therapy: international multidisciplinary consensus recommendations. International Wound Journal. 2016;14:385-98.

36. American College of Obstetricians and Gynecologists (ACOG). Use of Prophylactic Antibiotics in Labor and Delivery. 2018.

37. Liu Z, Dumville J, Norman G, Westby M, Blazeby J, McFarlane E, et al. Intraoperative interventions for preventing surgical site infection: an overview of Cochrane Reviews. Cochrane Database of Systematic Reviews. 2018(Issue 2. Art. No.: CD012653.).

38. Temming LA, Raghuraman N, Carter EB, Stout MJ, Rampersad RM, Macones GA, et al. Impact of evidence-based interventions on wound complications after cesarean delivery. American Journal of Obstetrics and Gynecology. 2017;217(4):449.e1-.e9.

39. Henman K, Gordon C, Gardiner T, Thorn J, Spain B, Davies J, et al. Surgical site infections following caesarean section at Royal Darwin Hospital, Northern Territory Infection, Disease and Health. 2012;17(2):47-51.

40. Kunkle C, Marchan J, Safadi S, Whitman S, Chmait R. Chlorhexidine gluconate versus povidone iodine at cesarean delivery: a randomized controlled trial. Journal of Maternal-Fetal \& Neonatal Medicine. 2014;28(5):573-7.

41. Gunatilake R, Swamy G, Brancazio L, Smrtka M, Thompson J, Gilner J, et al. Closed-Incision Negative-Pressure Therapy in Obese Patients Undergoing Cesarean Delivery: A Randomized Controlled Trial. American Journal of Perinatology Reports. 2017;7(3):e151-e7.

42. Hyldig N, Vinter C, Kruse M, Mogensen O, Bille C, Sorensen J, et al. Prophylactic incisional negative pressure wound therapy reduces the risk of surgical site infection after caesarean section in obese women: a pragmatic randomised clinical trial. British Journal of Obstetricians and Gynaecologists. 2018. 
43. Yu L, Kronen R, Simon L, Stoll C, Colditz G, G. Tuuli M. Prophylactic negative-pressure wound therapy after cesarean is associated with reduced risk of surgical site infection: a systematic review and meta-analysis. American Journal of Obstetrics \& Gynecology. 2017;18(2):200-10.

44. Scheck S, Blackmore T, Maharaj D, Langdana F, Elder R. Caesarean section wound infection surveillance: information for action. Australian and New Zealand Journal of Obstetrics and Gynaecology. 2018;58(5):518-24.

45. Ward V, Charlett A, Fagan J, Crawshaw S. Enhanced surgical site infection surveillance following caesarean section: experience of a multicentre collaborative post-discharge system. Journal of Hospital Infection. 2008;70(2):166-73.

46. Noy D, Creedy D. Postdischarge surveillance of surgical site infections: a multi-method approach to data collection. American Journal of Infection Control. 2002;30(7):417-24.

\section{Tables}

Due to technical limitations, tables are only available as a download in the supplemental files section.

\section{Figures}




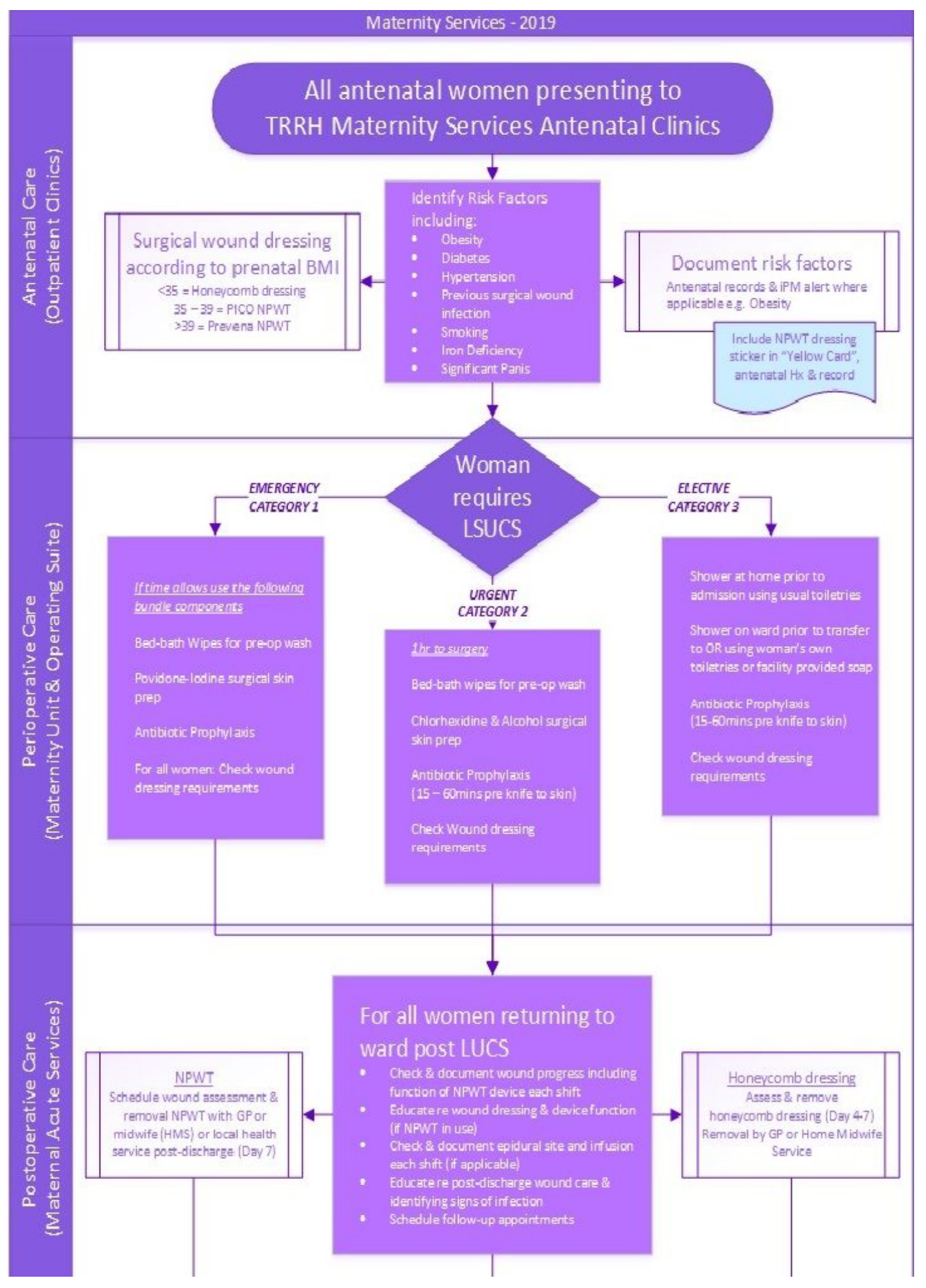

Figure 1

"CIP" Bundle Risk Assessment Algorithm 


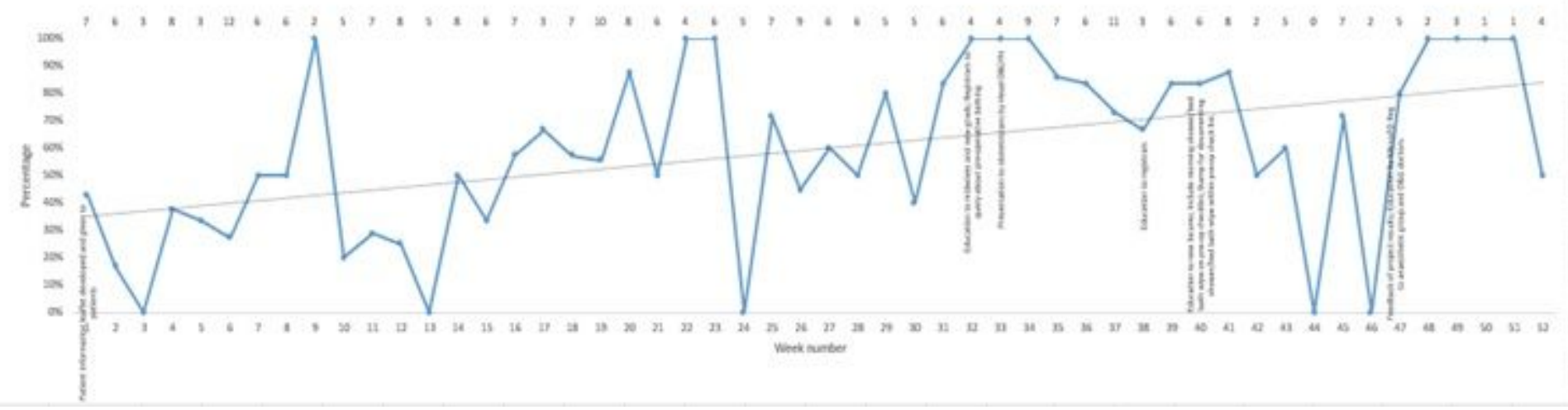

Figure 2

Percent of patients who received pre-operative site skin preparation

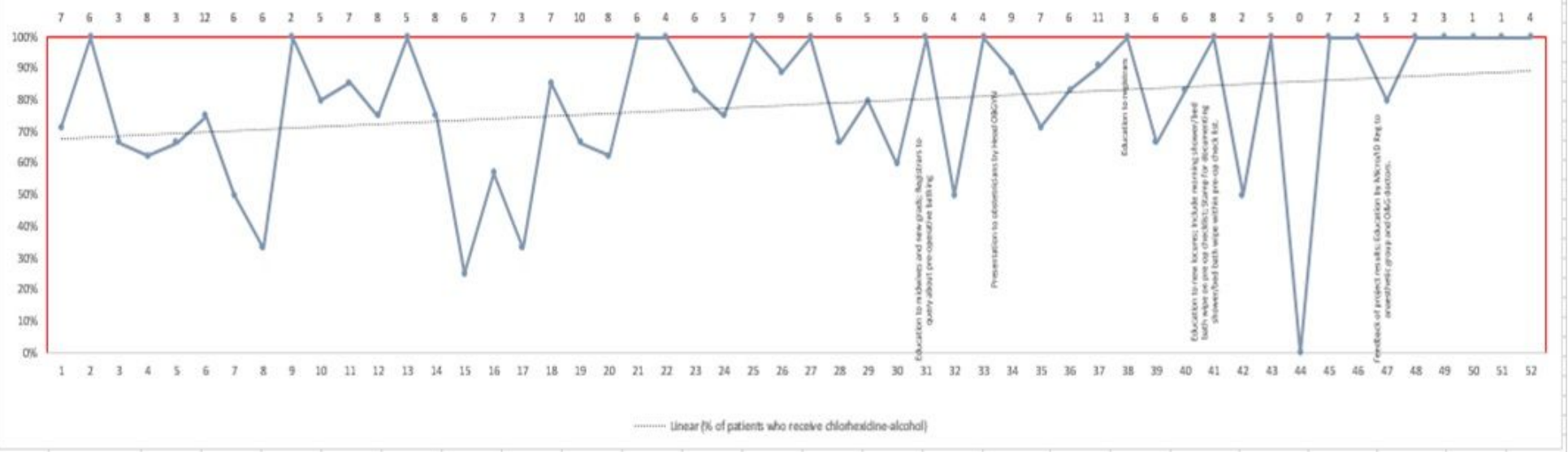

Figure 3

Percent of patients who received operative site preparation with Chlorhexidine and alcohol

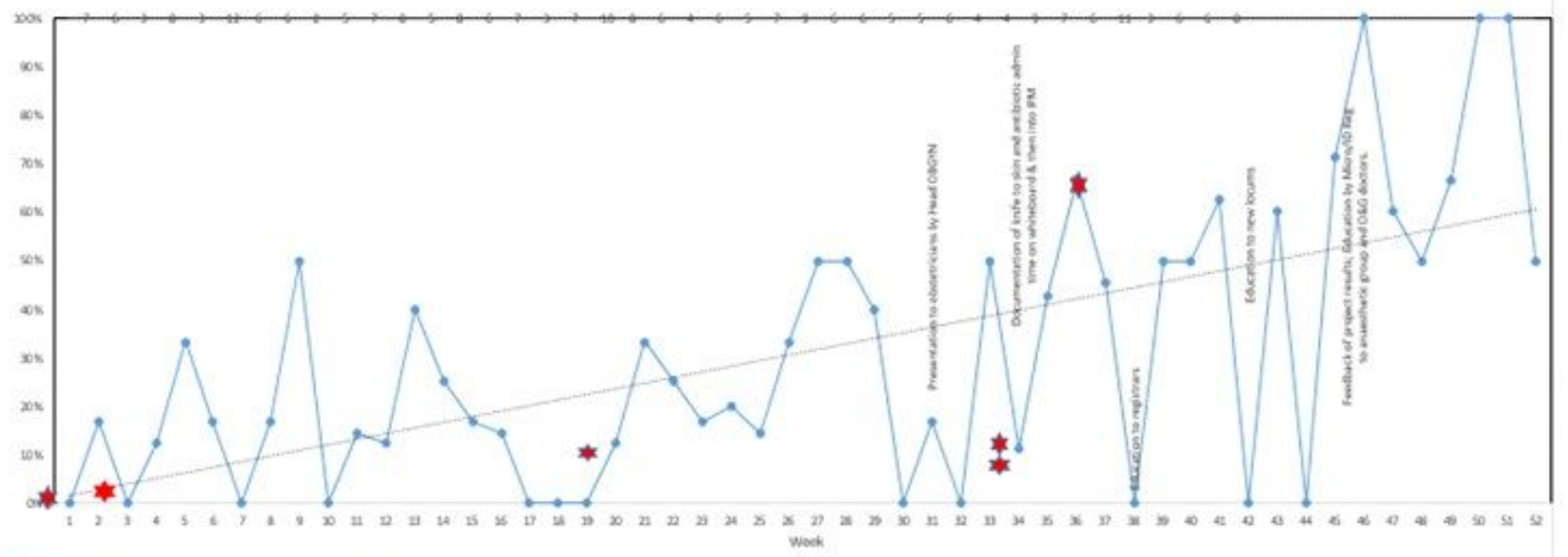

Indicates surgical site infection

Figure 4 
Percent of patients who received antibiotics prophylaxis 15-60 minutes pre-incision

\section{Supplementary Files}

This is a list of supplementary files associated with this preprint. Click to download.

- Tables17.pdf 